\title{
miR-183-5p attenuates cerebral ischemia injury by negatively regulating PTEN
}

\author{
LI ZHU ${ }^{1,2 *}$ XUEYING ZHOU ${ }^{3 *}$, SHANSHAN LI ${ }^{2}$, JIANMENG LIU ${ }^{4}$, \\ JINGYAN YANG $^{5}$, XIANGYUN FAN ${ }^{6}$ and SHENGNIAN ZHOU ${ }^{1}$
}

\begin{abstract}
${ }^{1}$ Department of Neurology, Qilu Hospital of Shandong University and Brain Science Research Institute, Shandong University, Jinan, Shandong 250012; ${ }^{2}$ Department of Neurology, Binzhou People's Hospital, Binzhou, Shandong 256610;

${ }^{3}$ Department of Rehabilitation, Qilu Hospital of Shandong University, Jinan, Shandong 250012; ${ }^{4}$ Department of Gynaecology and Obstetrics, Binzhou People's Hospital, Binzhou, Shandong 256610; ${ }^{5}$ Department of Pathology, The Second Hospital of

Shandong University, Jinan, Shandong 250033; ${ }^{6}$ Department of General Medicine,

Binzhou People's Hospital, Binzhou, Shandong 256610, P.R. China
\end{abstract}

Received December 20, 2019; Accepted July 28, 2020

DOI: $10.3892 / \mathrm{mmr} .2020 .11493$

\begin{abstract}
Cerebral ischemia is a common cerebrovascular disease caused by the occlusion of a cerebral blood vessel. MicroRNAs (miRNAs/miRs) are emerging regulators of various human diseases, including cerebral ischemia. Upregulation of miR-183-5p has been reported to alleviate liver injury induced by ischemia-reperfusion (I/R). However, the effect of miR-183-5p on cerebral ischemia injury remains unknown. The present study evaluated the effects of miR-183-5p on ischemia injury using ischemic models of mouse brains exposed to transient middle cerebral artery occlusion and Neuro-2A (N2A) neuroblastoma cells exposed to oxygen-glucose-deprivation (OGD) and subsequently reoxygenated. Ischemia was evaluated in mice using neurological function scores, cerebral edema, 2,3,5-triphenyltetrazoliumchloride, Nissl and Fluoro-Jade B staining assays. In addition, miR-183-5p expression, N2A cell viability and the expression levels of apoptosis-associated proteins were detected by quantitative PCR, Cell Counting Kit- 8 assay, flow cytometry and western blotting. The association between miR-183-5p and phosphatase and tensin homolog (PTEN) was also confirmed by a luciferase reporter assay. The results revealed that miR-183-5p expression was decreased and brain damage was increased in ischemic mice compared with
\end{abstract}

Correspondence to: Professor Shengnian Zhou, Department of Neurology, Qilu Hospital of Shandong University and Brain Science Research Institute, Shandong University, 107 West Wenhua Road, Jinan, Shandong 250012, P.R. China

E-mail: zhou_shengnian@126.com

*Contributed equally

Key words: cerebral ischemia, middle cerebral artery occlusion, microRNA-183-5p, oxygen-glucose-deprivation, phosphatase and tensin homolog the sham group. Additionally, miR-183-5p levels were reduced, and apoptosis was increased in N2A cells exposed to ischemia compared with the control group. Following transfection with agomiR-183-5p, cerebral ischemic injury and apoptosis levels were reduced in the in vivo I/R stroke model and OGD-induced $\mathrm{N} 2 \mathrm{~A}$ cells. In addition, PTEN was determined to be a target of miR-183-5p following elucidation of a direct binding site. Overexpression of PTEN reversed the miR-183-5p-induced N2A cell apoptosis inhibition and survival after OGD. The results of the present study suggested that miR-183-5p reduced ischemic injury by negatively regulating PTEN, which may aid the development of a novel therapeutic strategy for cerebral ischemia.

\section{Introduction}

Ischemic stroke refers to a type of cerebrovascular disease caused by the occlusion of a cerebral blood vessel, disrupting the supply of nutrients and oxygen to the brain and ultimately leading to brain tissue necrosis and neurological damage (1). As the second leading cause of death in industrialized countries, ischemic injury accounts for $87 \%$ of all strokes (2). It has been reported that $\sim 20 \%$ of stroke survivors need institutional care, and $15-30 \%$ of stroke survivors remain permanently disabled (3). Intravenous recombinant tissue plasminogen activator is an effective treatment of ischemia; however, only $5 \%$ patients are eligible for treatment, and when administered for $>4.5 \mathrm{~h}$, the risk of intracranial hemorrhage may exceed its benefit (4). Previous studies have demonstrated that cerebral ischemia may lead to apoptosis and neuronal damage in the ischemic area (5), although the subtle underlying mechanisms are not fully understood. Therefore, clarifying the molecular mechanism of cerebral ischemia may provide new insights into ischemic stroke. MicroRNAs (miRNAs/miRs) are small non-coding single-stranded RNA molecules $\sim 22$ nucleotides long that regulate gene expression after transcription by inhibiting translation or inducing the degradation of the target gene mRNA (6). In recent years, increasing studies have suggested 
that miRNAs serve vital roles in ischemic stroke (7). In addition, experimental therapies based on miRNAs have been developed to assist post-stroke neurological recovery and mitigate ischemic brain injury $(8,9)$. Chang et al (10) have reported that miR-195 ameliorates ischemic stroke through inhibiting neuronal apoptosis in a middle cerebral artery occlusion (MCAO) model of brain ischemia. miR-183, which belongs to the evolutionarily conserved miRNA cluster, is located on human chromosome 7 and serves a number of functions in key cellular processes, including the development of neurons (11). Lin et al (12) have demonstrated that ischemic post-conditioning increases miR-183-5p levels in the murine liver compared with those in ischemia-reperfusion (I/R) mice. Furthermore, miR-183-5p upregulation alleviates liver injury after I/R by targeting apoptotic protease-activating factor 1. Gong et al (13) have reported that knockdown of long non-coding RNA maternally expressed 3 upregulates the levels of miR-183-5p, thus protecting H9c2 rat cardiomyocytes from hypoxic injury. However, to the best of our knowledge, whether miR-183-5p mitigates cerebral ischemia injury has not been reported.

The phosphatase and tensin homolog (PTEN) gene, located on human chromosome 19, encodes a dual protein phosphatase enzyme that dephosphorylates protein and lipid substrates (14). A previous study has revealed increased expression levels of PTEN in MCAO or oxygen-glucose deprivation (OGD)-induced ischemic stroke (15). In addition, knockdown of miR-183-5p upregulated PTEN expression in multiple types of tumor (including synovial sarcoma, rhabdomyosarcoma and colon cancer) and $\mathrm{T}$ cell-dependent autoimmune diseases $(16,17)$. Based on these findings, the current study hypothesized that miR-183-5p may reduce cerebral ischemic injury by regulating PTEN. This hypothesis was verified by inducing transient focal cerebral ischemia in mice exposed to MCAO and exposing Neuro-2A neuroblastoma (N2A) cells to OGD.

\section{Materials and methods}

Animal model of focal cerebral ischemia. In the present study, a total of 112 male 8-week-old C57BL/6 mice were purchased from Changsheng Bio-technology Co., Ltd. All mice were maintained in a controlled environment with a temperature of $21-23^{\circ} \mathrm{C}$ and a humidity of $45-55 \%$ under a 12 -h light/dark cycle with free access to food and water. After adaptive feeding for 1 week, mice were randomly divided into the sham group $(n=32)$ and the experimental group $(n=80)$. Animals in the experimental group were subjected to cerebral ischemia using the MCAO method as previously described (18). Mice were anesthetized with $50 \mathrm{mg} / \mathrm{kg}$ pentobarbital sodium. The common carotid artery and the vagus nerve were exposed by a midline neck incision. Then, the internal carotid artery (ICA) and external carotid artery (ECA) were bluntly isolated. After a small opening was made between the ligation (at the distal end of ECA) and the clipping (at the proximal end of ECA and CCA), a plug (diameter, 0.22-0.23 mm) was inserted to block the blood flow of the middle cerebral artery, followed by suturing of the skin. The plug was pulled out $1 \mathrm{~h}$ after ischemia, and subsequent experiments were performed $24 \mathrm{~h}$ after reperfusion. For the sham surgery, all procedures were identical, with the exception that the plug was not inserted. All procedures using laboratory animals were in accordance with the Institutional Animal Care and Use Committee of Binzhou People's Hospital (approval no. LYP200).

The mice were randomly divided into four groups: i) Sham ( $\mathrm{n}=32)$; ii) MCAO (n=32); iii) MCAO + agomiR-183-5p ( $\mathrm{n}=24)$; and iv) $\mathrm{MCAO}+$ agomiR-negative control (NC) $(\mathrm{n}=24)$. In each group, mice were used to evaluate mRNA and protein expression $(n=8)$, determine the cerebral infarct area $(n=8)$, evaluate cerebral edema $(n=8)$ and determine neurological scores by histopathological analysis of the ischemic penumbra $(n=8)$.

AgomiR-183-5p transfection into the mouse brain. The miR-183-5p agomir (agomiR-183-5p; 5'-UAUGGCACUGGU AGAAUUCACU-3') and its negative control (agomiR-NC; 5'-UUCUCCGAACGUGUCACGUTT-3') were obtained from Shanghai GenePharma Co., Ltd. In vivo transfection was performed as previously described (19): The stereotaxic coordinates were $0.5 \mathrm{~mm}$ posterior and $1.0 \mathrm{~mm}$ lateral to the bregma, and 2.5-3.0 mm ventral to the surface of the skull. To assess the neurological dysfunction and neuronal damage in mice following MACO, agomiR-183-5p or agomiR-NC was mixed with the Entranster ${ }^{\mathrm{TM}}$-in vivo transfection reagent (Engreen Biosystem Co., Ltd.) and subsequently injected intracerebroventricularly into the experimental group mice (the $\mathrm{MCAO}+$ agomiR-183-5p and $\mathrm{MCAO}+$ agomiR-NC groups) at a rate of $0.2 \mu \mathrm{l} / \mathrm{min}$ using a mini-pump prior to cerebral ischemia. For the MCAO group, an equal amount of Entranster $^{\mathrm{TM}}$-in vivo was injected. After $24 \mathrm{~h}$, cerebral ischemia was induced in the experimental group via MCAO.

Neurological scoring. Neurological deficits were evaluated immediately after reperfusion to verify the MCAO model. Deficits were scored using an 18-point scoring system as previously described (20). The scoring system was six parts: i) Spontaneous activity in the cage for $5 \mathrm{~min}$; ii) limb activity symmetry; iii) forelimb symmetry; iv) climbing in metal wire cages; v) torso touching; and vi) vibrissa responses. The scores of each test ranged between 0 and 3, with a score of 0 indicating a normal neurological response.

Brain edema assay. Mice were sacrificed under deep anesthesia (150 mg/kg pentobarbital sodium; death was confirmed by lack of breathing and heartbeat) at $24 \mathrm{~h}$ post-reperfusion, and the brain tissue was harvested. Brain edema was analyzed using the wet/dry method as previously described (21). Brain water content was calculated using the following formula: [(wet weight-dry weight)/wet weight] x100\%.

\section{2,3,5-Triphenyltetrazoliumchloride (TTC) staining. The} area of cerebral infarction was measured as previously described (22). Brain tissues from mice were frozen at $-20^{\circ} \mathrm{C}$ for $1-2 \mathrm{~h}$. The olfactory bulb and the cerebellum were removed, and the tissues were sliced into five 1-mm thick coronal sections and stored on ice. The sections were immersed in 1\% TTC (Shanghai Aladdin Bio-chem Technology Co., Ltd.) and stained for $10-15 \mathrm{~min}$ at $37^{\circ} \mathrm{C}$ in the dark, during which the slices were continuously turned to ensure even coverage. The cerebral infarct area was photographed using a digital camera and quantified by Image-pro plus 6.0 software (Media Cybernetics, Inc.). 
Nissl staining. Samples of the ischemic penumbra were obtained from mice $24 \mathrm{~h}$ after reperfusion. Neuronal damage in the ischemic penumbra was assessed using Nissl staining as previously described (23). Samples were fixed with $4 \%$ paraformaldehyde for $15 \mathrm{~min}$ at room temperature, embedded in paraffin, dewaxed in xylene and rehydrated with graded ethanol $(95,85$ and $75 \%)$. The samples were cut coronally with a thickness of $4 \mu \mathrm{m}$. Following rinsing with distilled water, the sections were stained with $0.5 \%$ crystal violet (Sinopharm Chemical Reagent Co., Ltd.) for $10 \mathrm{~min}$ at room temperature. Images of the ischemic penumbra were observed under an Olympus BX53 light microscope (Olympus Corporation) at x200 magnification. The number of neurons was quantified by a professional researcher, who was blind to the grouping.

Fluoro-Jade B (FJB) staining. FJB staining was performed using an FJB staining kit (EMD Millipore) according to the manufacturer's instructions. Coronary sections $(4 \mu \mathrm{m})$ from the ischemic penumbra were visualized using an Olympus BX53 fluorescence microscope at magnification, x200 (Olympus Corporation) to evaluate degenerating neurons. FJB-positive neurons were counted by a professional researcher, who was blind to the grouping.

Cell culture. Mouse N2A cells were purchased from Procell Life Science \& Technology Co., Ltd. and cultured in Dulbecco's Modified Eagle's Medium (DMEM; Thermo Fisher Scientific, Inc.) containing high glucose (HG) and $10 \%$ fetal bovine serum (Thermo Fisher Scientific, Inc.) at $37^{\circ} \mathrm{C}$ in a humidified atmosphere of $5 \% \mathrm{CO}_{2}$.

$O G D$ and reoxygenation. To simulate ischemic-like conditions in vitro, N2A cells were seeded on 6-well plates $\left(4 \times 10^{5}\right.$ cells/well) and incubated at $37^{\circ} \mathrm{C}$ with $5 \% \mathrm{CO}_{2}$ for $24 \mathrm{~h}$. Subsequently, $\mathrm{HG}$ medium was replaced with glucose-free DMEM and incubated at $37^{\circ} \mathrm{C}$ in an anaerobic chamber containing $95 \% \mathrm{~N}_{2}$ and $5 \% \mathrm{CO}_{2}$ for $3 \mathrm{~h}$. Following OGD exposure, cells were returned to $\mathrm{HG}$ medium under normoxic conditions for 24-h reoxygenation. Control cells not exposed to OGD were maintained in DMEM containing $\mathrm{HG}$ at $37^{\circ} \mathrm{C}$ with $5 \% \mathrm{CO}_{2}$ and $95 \% \mathrm{O}_{2}$.

Cell transfection. N2A cells were plated into a 6-well plate $\left(4 \times 10^{5}\right.$ cells/well), cultured overnight, and were then transfected with 100 pmol agomiR-183-5p, antagomiR-183-5p or their negative controls at room temperature for $48 \mathrm{~h}$ using Lipofectamine $^{\circledR} 2000$ (Invitrogen; Thermo Fisher Scientific, Inc.) according to the manufacturer's instructions. Additionally, a PTEN-overexpression pcDNA3.1 plasmid (1 $\mu \mathrm{g}$; Invitrogen; Thermo Fisher Scientific, Inc.) was transfected alone or co-transfected with 50 pmol agomiR-183-5p into N2A cells using Lipofectamine ${ }^{\circledR} 2000$ (Invitrogen; Thermo Fisher Scientific, Inc.) according to the manufacturer's instructions. An empty pcDNA3.1 vector was used as a negative control. OGD was performed $48 \mathrm{~h}$ after transfection.

Luciferase activity assay. TargetScan (http://www.targetscan. org) was used to predict the potential targets of miR-183-5p, and an interaction between miR-183-5P and PTEN was identified. N2A cells were incubated in 12-well plates at $90 \%$ confluence for $24 \mathrm{~h}$. Cells were then co-transfected with the pMIR-reporter luciferase vector, which included the wild-type or mutant PTEN-3'-untranslated region (UTR) miR-183-5p binding site 1 or 2 and agomiR-NC or agomiR-183-5p using Lipofectamine ${ }^{\circledR} 2000$ (Invitrogen; Thermo Fisher Scientific, Inc.) according to the manufacturer's instructions. After 48-h transfection, luciferase activity was detected by the Luciferase Assay kit (Promega Corporation) according to the manufacturer's instructions. Luciferase activity was normalized to the Renilla luciferase activity.

Reverse transcription-quantitative $(R T-q) P C R$. Total RNA was extracted from the ischemic penumbra or N2A cells using the RNApure kit (BioTeke Corporation) according to the manufacturer's instructions. To determine miR-183-5p or PTEN mRNA expression, reverse transcription was performed using M-MLV Reverse Transcriptase (2641A, Takara Biotechnology Co., Ltd.) according to the manufacturer's directions. Real-time PCR was subsequently conducted using Taq $^{\mathrm{TM}}$ HS Perfect Mix (Takara Biotechnology Co., Ltd.) and SYBR ${ }^{\circledR}$ Green (BioTeke Corporation). The forward and reverse primers of miR-183-5p, U19, PTEN and $\beta$-actin used for real-time PCR were as follows: miR-183-5p, 5'-GCG GCTATGGCACTGGTAGAA-3' and 5'-GTGCAGGGTCCG AGGTATTC-3'; U19, 5'-TGTGGAGTTGGTCCTGGTCT-3' and 5'-GTGCAGGGTCCGAGGTATTC-3'; PTEN, 5'-GAC CATAACCCACCACAGC-3' and 5'-CATTACACCAGTCCG TCCCT-3'; $\beta$-actin, 5'-AATCGTGCGTGACATCAA-3' and 5'-AGAAGGAAGGCTGGAAAA-3'. Relative miR-183-5p was calculated using the $2^{-\triangle \Delta \mathrm{Cq}}$ method (24) and normalized to U19. Relative PTEN expression was calculated using the same method and normalized to $\beta$-actin.

Cell Counting Kit-8 (CCK-8) assay. Cell viability was assessed using a CCK-8 kit (Sigma-Aldrich; Merck KGaA) 24 h after OGD according to the manufacturer's instructions. Briefly, N2A cells from different groups, including i) Control; ii) OGD; iii) OGD + agomiR-NC; iv) OGD + agomiR-183-5p; v) OGD + agomiR-183-5p + vector; and vi) OGD + agomiR-183-5p + PTEN-overexpression plasmid, were plated in 96-well plates $\left(3 \times 10^{3}\right.$ cells/well). Following the addition of $\mathrm{CCK}-8$ solution to each well, the cells were incubated for $2 \mathrm{~h}$ at $37^{\circ} \mathrm{C}$ with $95 \% \mathrm{O}_{2}$ and $5 \% \mathrm{CO}_{2}$. Optical density values were measured at $450 \mathrm{~nm}$ using an ELX-800 microplate reader (BioTeke Corporation).

Flow cytometry assay. OGD-induced apoptosis was detected using an Annexin-V/propidium iodide (PI) apoptosis detection kit (Beyotime Institute of Biotechnology) according to the manufacturer's instructions. Briefly, N2A cells from each group were harvested after centrifugation at $90 \mathrm{x} \mathrm{g}$ for $5 \mathrm{~min}$ at room temperature and washed twice with PBS. Next, the cells were stained with Annexin V-FITC and PI for $15 \mathrm{~min}$ in the dark. Finally, apoptosis was analyzed using a flow cytometer (NovoCyte, Aceabio Biosciences, Inc.) and NovoExpress software version 1.3.1 (Aceabio Biosciences, Inc.).

Western blotting. Total protein was extracted from the ischemic penumbra or N2A cells and quantified using a BCA kit (Beyotime Institute of Biotechnology) according to the manufacturer's instructions. Equal amounts of protein 
(30 $\mu \mathrm{g}$ per lane) were separated on $10-15 \%$ sodium dodecyl sulfate-polyacrylamide gels and transferred to polyvinylidene fluoride membranes (Thermo Fisher Scientific, Inc.). The membranes were blocked with $5 \%(\mathrm{~m} / \mathrm{v})$ bovine serum albumin (Biosharp Life Sciences) for $1 \mathrm{~h}$ at room temperature and incubated at $4^{\circ} \mathrm{C}$ overnight with the following primary antibodies: Anti-B-cell lymphoma-extra-large (Bcl-xl; 1:1,000; cat. no. 2762; Cell Signaling Technology, Inc.), anti-B-cell lymphoma-2 (Bcl-2; 1:500; cat. no. 12789-1-AP; ProteinTech Group, Inc.), anti-Bcl-2-associated X protein (Bax; 1:500; cat. no. 50599-2-Ig; ProteinTech Group, Inc.), anti-cleaved caspase-3 (1:1,000; cat. no. 9654; Cell Signaling Technology, Inc.), anti-pro caspase-3 (1:1,000; cat. no. 9662; Cell Signaling Technology, Inc.), anti-PTEN (1:1,000; cat. no. A11193; ABclonal Biotech Co., Ltd.) or anti- $\beta$-actin (1:2,000; cat. no. 60008-1-Ig; ProteinTech Group, Inc.). After washing with TBS $+0.1 \%$ Tween-20, the membranes were incubated for $40 \mathrm{~min}$ at $37^{\circ} \mathrm{C}$ with horseradish peroxidase-labeled goat anti-rabbit $\mathrm{IgG}$ (1:10,000; cat. no. SA00001-2; ProteinTech Group, Inc.) or goat anti-mouse IgG (1:10,000; cat. no. SA00001-1; ProteinTech Group, Inc.) secondary antibodies. The signal bands of the membranes were visualized using an ECL kit (Shanghai 7sea Biotech Co., Ltd.) according to the manufacturer's protocol and quantified by Gel-Pro-Analyzer 4.0 software (Media Cybernetics, Inc.).

Statistical analysis. Data are presented as the mean $\pm \mathrm{SD}$, and each experiment was repeated $\geq 3$ times. Statistical analyses were performed by GraphPad Prism 8.0 (GraphPad Software, Inc.) using one-way ANOVA followed by Tukey's test for multiple comparisons or two-tailed unpaired Student's t-tests for comparisons between two groups. $\mathrm{P}<0.05$ was considered to indicate a statistically significant difference.

\section{Results}

Expression of miR-183-5p in the MCAO mouse model. To identify the expression of miR-183-5p in vivo before and after ischemia induction, mice were subjected to MCAO $(\sim 25 \%$ mortality). The results of the RT-qPCR assay demonstrated that the expression of miR-183-5p was significantly decreased after MCAO ( $\mathrm{P}<0.05$; Fig. 1A) compared with that in the sham group, indicating that downregulation of miR-183-5p expression in vivo was associated with cerebral ischemia.

Effects of miR-183-5p on the neurological score, cerebral edema and infarct area. To explore the role of miR-183-5p on cerebral ischemia, agomiR-NC or agomiR-183-5p was injected into the murine cerebra prior to MCAO. RT-qPCR analysis revealed that compared with agomiR-NC, transfection with agomiR-183-5p significantly upregulated the expression of miR-183-5p in the MCAO group $(\mathrm{P}<0.05$; Fig. 1B). Neurological score and brain water content analyses were performed to assess the neurological function and brain edema, respectively, and TTC staining was performed to determine the area of cerebral infarction. As demonstrated in Fig. $1 \mathrm{C}$, the neurological scores of the mice in the MCAO group were markedly increased compared with those of mice in the sham group. Additionally, agomiR-183-5p significantly decreased the neurological scores compared with those in the
$\mathrm{MCAO}+$ agomiR-NC group $(\mathrm{P}<0.05)$. As demonstrated in Fig. 1D-F, brain water content and infarct area (demonstrated as white coloration) were significantly increased in the MCAO group compared with that in the sham group, and these effects were relieved by agomiR-183-5p compared with those in the $\mathrm{MCAO}+$ agomiR-NC group $(\mathrm{P}<0.05)$.

Effects of miR-183-5p on neuronal damage and apoptosis. To further study the extent of neuronal damage, Nissl and FJB staining of brain tissues was performed. The results of Nissl staining demonstrated that the number of normal neurons was markedly decreased following MCAO treatment compared with that in the sham-treated mice; however, the numbers were increased after injecting mice with agomiR-183-5p compared with that in the $\mathrm{MCAO}+$ agomiR-NC group $(\mathrm{P}<0.05$; Fig. $2 \mathrm{~A}$ and $\mathrm{B})$. Consistent with this result, the number of FJB-positive cells observed in the MCAO group was significantly higher compared with that in the sham group, but significantly reduced after injection with agomiR-183-5p ( $<<0.05$; Fig. 2C and D). In addition, the neuronal expression of apoptosis marker Bcl-xl was markedly inhibited and the levels of Bax and cleaved caspase-3 were increased in the MCAO group compared with that in the sham group; however, agomiR-183-5p reversed the MACO-induced expression of apoptosis-related proteins $(\mathrm{P}<0.05$; Fig. 2E). These results suggested that miR-183-5p effectively alleviated the brain damage caused by cerebral ischemia.

Expression of miR-183-5p in N2A cells exposed to OGD. To simulate ischemia-like conditions in vitro, N2A cells were exposed to OGD followed by reoxygenation. The in vitro expression of miR-183-5p before and after ischemia was detected by RT-qPCR. The results demonstrated that the expression of miR-183-5p after OGD treatment was significantly decreased compared with that in the control group $(\mathrm{P}<0.05$; Fig. 3A), suggesting that in vitro miR-183-5p expression was associated with ischemia.

Effects of miR-183-5p on the viability of $N 2 \mathrm{~A}$ cells exposed to $O G D$. To explore the role of miR-183-5p in N2A cells, agomiR-NC or agomiR-183-5p was transfected into N2A cells $48 \mathrm{~h}$ prior to OGD treatment. The expression levels of miR-183-5p in N2A cells was upregulated after transfection with agomiR-183-5p compared with that in the agomiR-NC group $(\mathrm{P}<0.05$; Fig. 3B). The viability of $\mathrm{N} 2 \mathrm{~A}$ cells exposed to OGD was subsequently measured using a CCK-8 assay. As presented in Fig. 3C, N2A cell viability was significantly decreased after exposure to OGD compared with that of the control cells, but transfection with agomiR-183-5p significantly increased N2A cell viability, indicating that miR-183-5p may promote cell survival $(\mathrm{P}<0.05)$.

Effects of miR-183-5p on apoptosis in N2A cells exposed to $O G D$. To analyze the apoptosis of $\mathrm{N} 2 \mathrm{~A}$ cells, flow cytometry and western blotting were carried out. The results of the flow cytometry assay demonstrated that the apoptotic rates in untreated N2A cells were low, whereas apoptosis was significantly increased following exposure to OGD. However, the increase in apoptosis was suppressed in N2A cells transfected with agomiR-183-5p compared with that in 

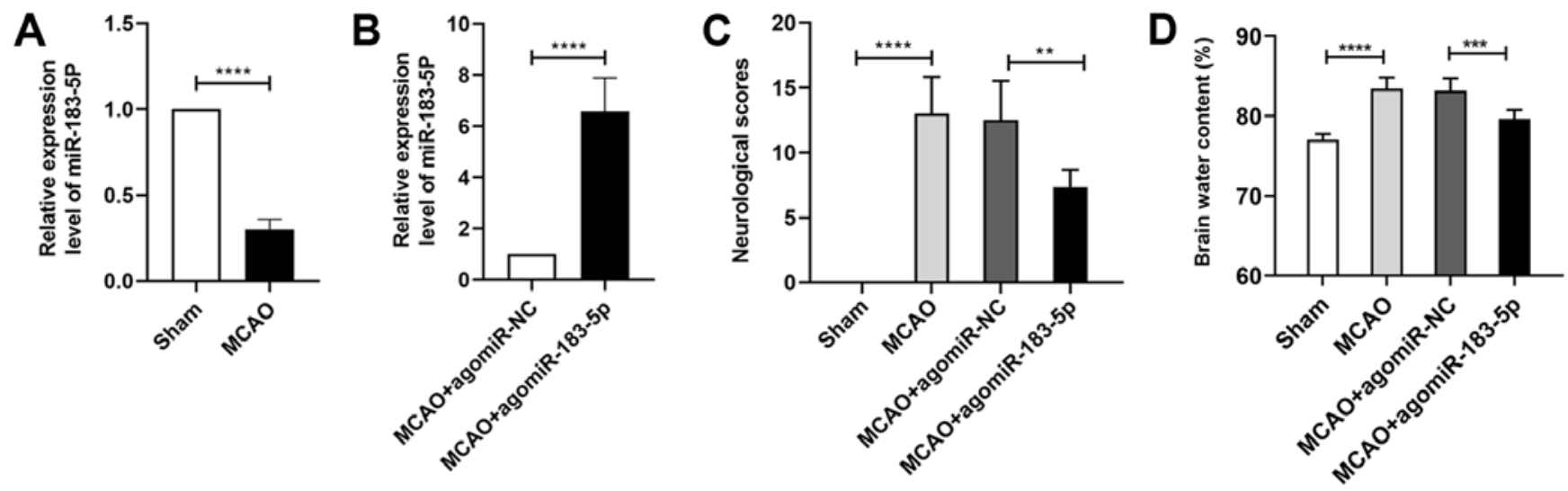

\section{$\mathbf{E}$}
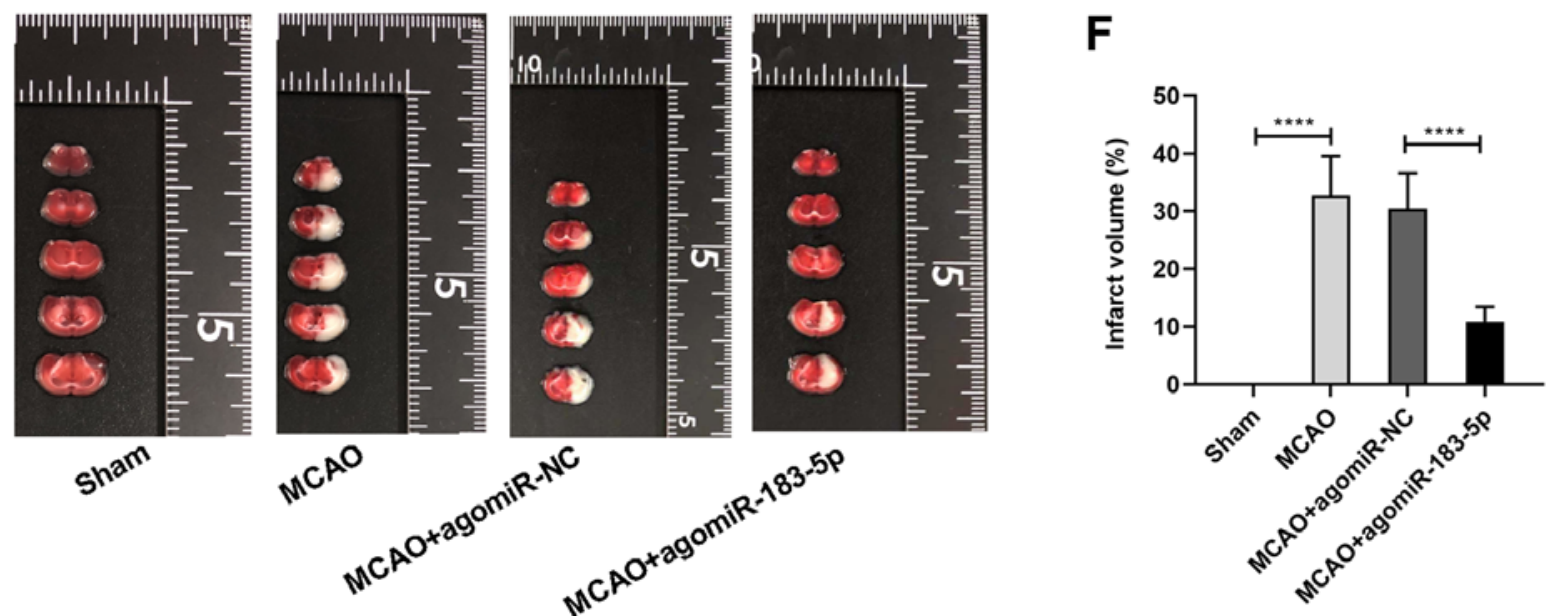

Figure 1. Effects of miR-183-5p on MCAO-induced ischemic injury in mouse brain. The mouse brain was injected with agomiR-NC or agomiR-183-5p before MCAO. (A) The relative expression level of miR-183-5p in the mouse brain was detected by reverse transcription-quantitative PCR. (B) The relative expression level of miR-183-5p in the ischemic penumbra after transfection with agomiR-183-5p. (C) The score of neurological function. (D) The edema detection of brain tissue. (E) The infarct volume of brain tissue evaluated by 2,3,5-triphenyltetrazoliumchloride staining. (F) The percentage of infarct volume in whole cerebral tissue. Data are expressed as the mean $\pm \mathrm{SD}(\mathrm{n}=8) .{ }^{* *} \mathrm{P}<0.01,{ }^{* * *} \mathrm{P}<0.001$ and ${ }^{* * * * *} \mathrm{P}<0.0001$. MCAO, middle cerebral artery occlusion; miR, microRNA; $\mathrm{NC}$, negative control.

cells transfected with agomiR-NC $(\mathrm{P}<0.05$; Fig. 3D and $\mathrm{E})$. The results of western blotting analysis demonstrated that the expression levels of the apoptosis-related markers Bax and cleaved caspase-3 were significantly upregulated in N2A cells exposed to OGD, and the expression levels of Bcl-2 upon OGD exposure were downregulated compared with those in the control group; transfection with agomiR-183-5p reversed these changes in apoptosis-related markers (Fig. $3 \mathrm{~F}$ and $\mathrm{G} ; \mathrm{P}<0.05$ ). These results suggested that miR-183-5p inhibited apoptosis in $\mathrm{N} 2 \mathrm{~A}$ cells.

PTEN is the target gene of miR-183-5p. To further elucidate the mechanism of miR-183-5p in cerebral ischemia-induced apoptosis, bioinformatics analysis was used to predict its target genes (http://www.targetscan.org). The results revealed that PTEN was predicted to be a target gene of miR-183-5p. Binding sites between PTEN-3'-UTR and miR-183-5p were elucidated, and their sequences are presented in Fig. 4A and B. To verify the interaction between miR-183-5p and PTEN, N2A cells were co-transfected with agomiR-NC or agomiR-183-5p and assessed by performing a luciferase reporter assay. As demonstrated in Fig. 4C and D, transfection with agomiR-183-5p significantly decreased the luciferase activity of the wild-type reporter gene containing PTEN-3'-UTR site
1 or $2(\mathrm{P}<0.05)$. No significant differences were observed in the luciferase activity of the mutated reporter gene containing PTEN-3'-UTR site 1 or 2 following agmiR-183-5p treatment. These results suggested that miR-183-5p could directly bind to the target gene PTEN.

In addition, to further determine the effects of miR-183-5p on PTEN, agomiR-NC, agomiR-183-5p, antagomiR-NC or antagomiR-183-5p was transfected into N2A cells. Transfection with antagomiR-183-5p decreased the levels of miR-183-5p in $\mathrm{N} 2 \mathrm{~A}$ cells compared with those in the antagomir-NC group $(\mathrm{P}<0.05$; Fig. 4E). The results of RT-qPCR and western blotting revealed that transfection with agomiR-183-5p significantly decreased PTEN levels, whereas antagomiR-183-5p significantly increased PTEN levels compared with those in the respective $\mathrm{NC}$ groups $(\mathrm{P}<0.05$; Fig. $4 \mathrm{~F}$ and $\mathrm{G})$. These results suggested that miR-183-5p inhibited PTEN protein expression by binding to its 3'-UTR.

PTEN overexpression partially reverses the effect of $m i R-183-5 p$. To determine the effect of miR-183-5p/PTEN signaling on OGD-treated N2A cells, a PTEN overexpression plasmid was transfected alone or co-transfected with agomiR-183-5p into N2A cells prior to OGD. The result of RT-qPCR demonstrated that transfection with the PTEN 
A
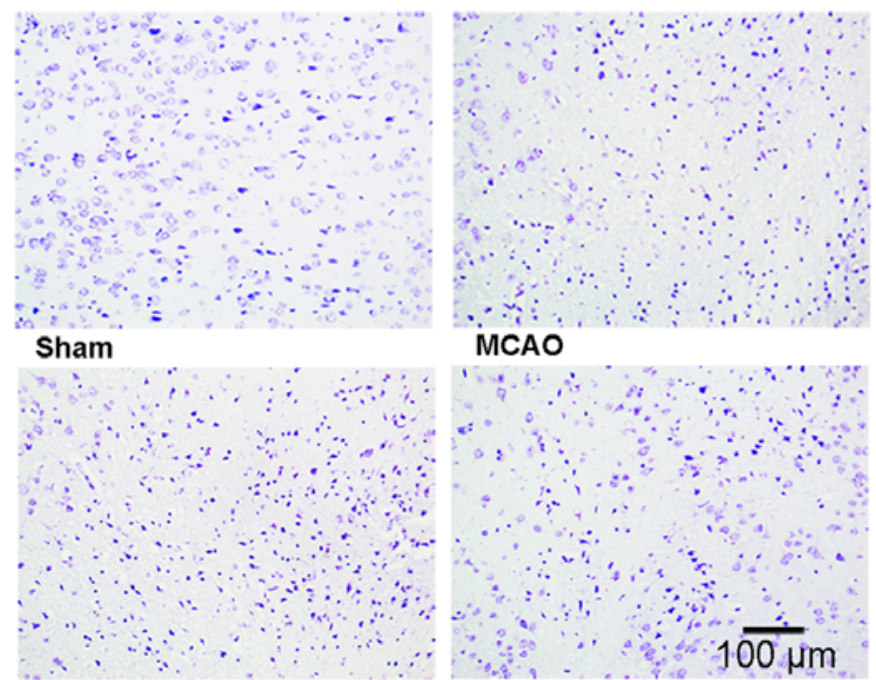

MCAO+agomiR-NC

C

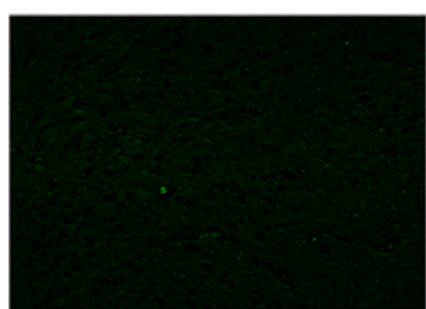

Sham

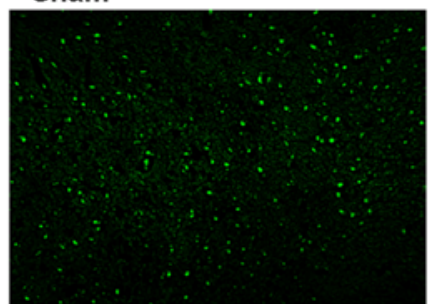

MCAO+agomiR-NC

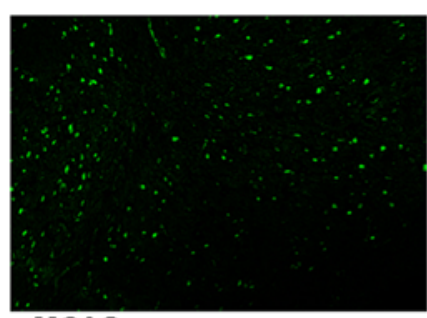

MCAO

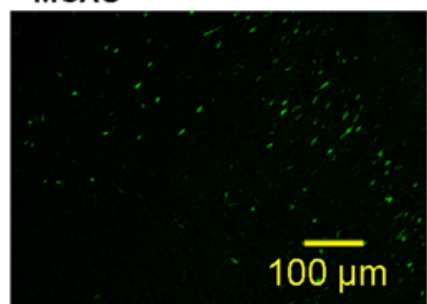

MCAO+agomiR-183-5p
B

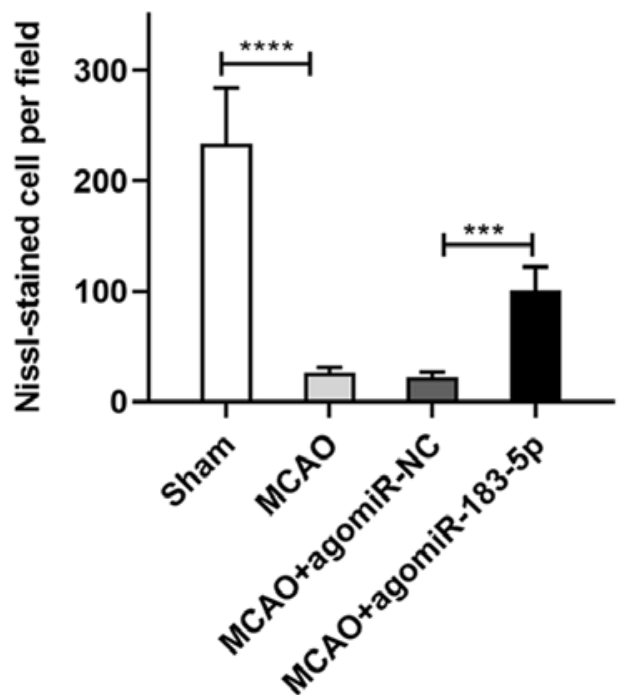

D

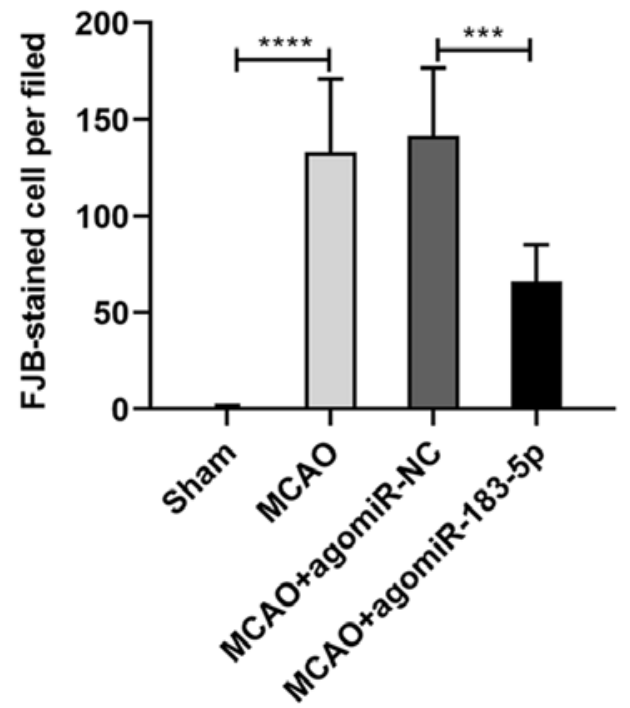

E

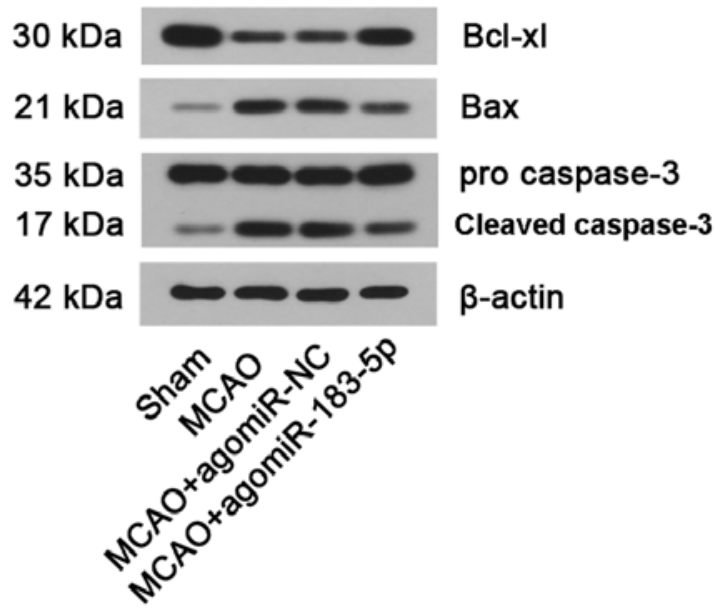

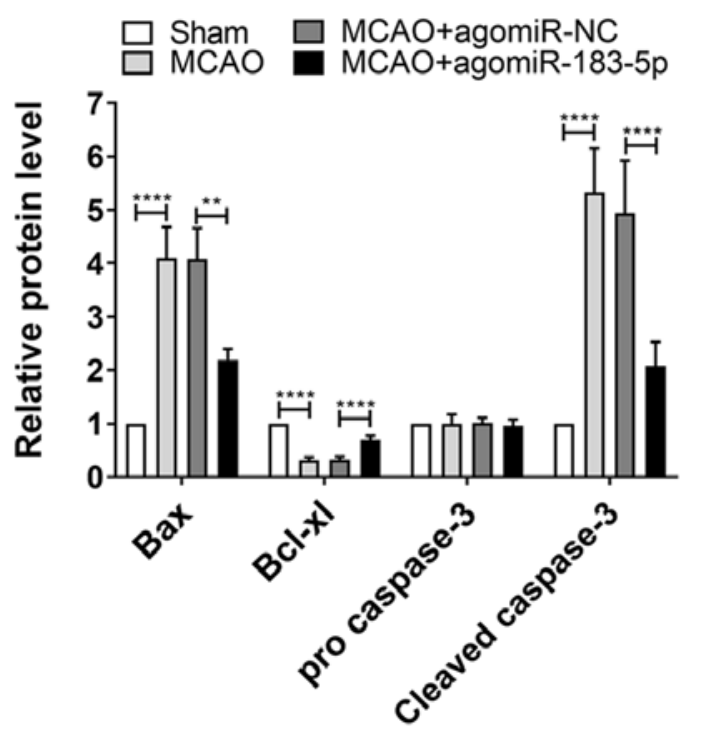

Figure 2. Analysis of neuronal damage at $24 \mathrm{~h}$ after reperfusion. The mice were injected with agomiR-NC or agomiR-183-5p before MCAO. (A) Normal neurons evaluated by Nissl staining. Scale bar, $100 \mu \mathrm{m}$. (B) Quantitative analysis of Nissl-stained cells. (C) Injured neurons evaluated by FJB staining. Scale bar, $100 \mu \mathrm{m}$. (D) Quantitative analysis of FJB-positive cells. (E) Relative protein expression levels of the Bax, Bcl-xl and cleaved caspase-3 in MCAO models of cerebral ischemia injury. Data are expressed as the mean $\pm \mathrm{SD}(\mathrm{n}=8) .{ }^{* *} \mathrm{P}<0.01,{ }^{* * *} \mathrm{P}<0.001$ and ${ }^{* * * * *} \mathrm{P}<0.0001$. MCAO, middle cerebral artery occlusion; miR, microRNA; NC, negative control; FJB, Fluoro-Jade B 
A

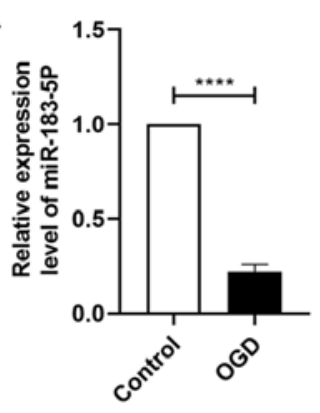

B

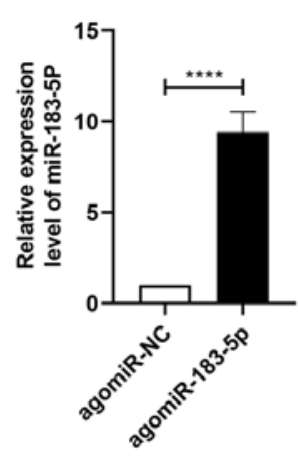

C

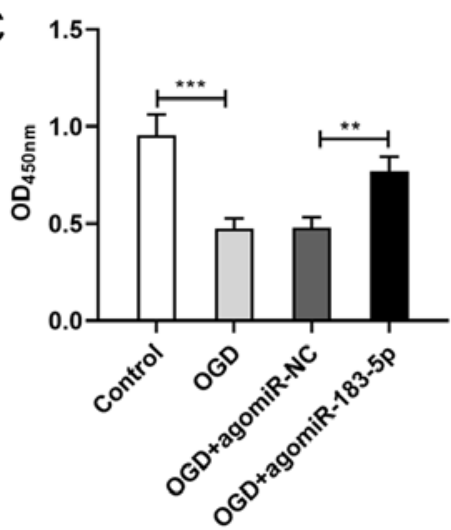

D $\underset{\pi}{z}$
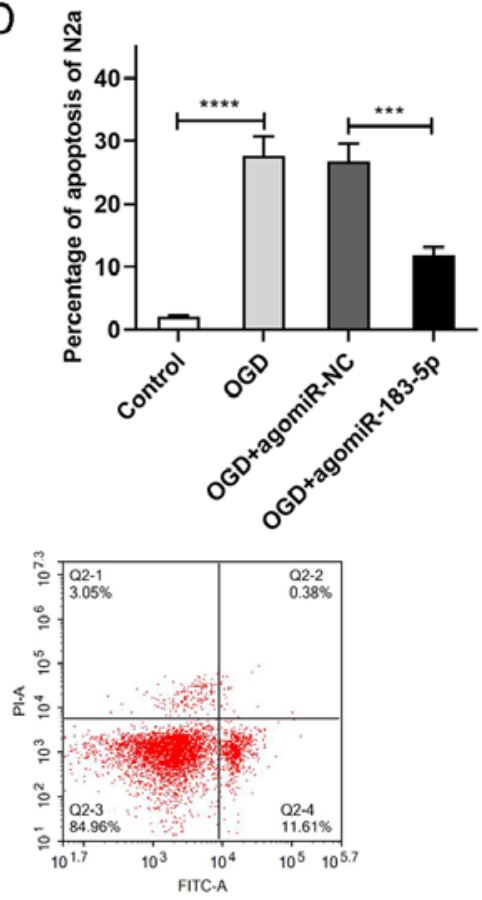

OGD+agomiR-183-5p
$\mathrm{F}$

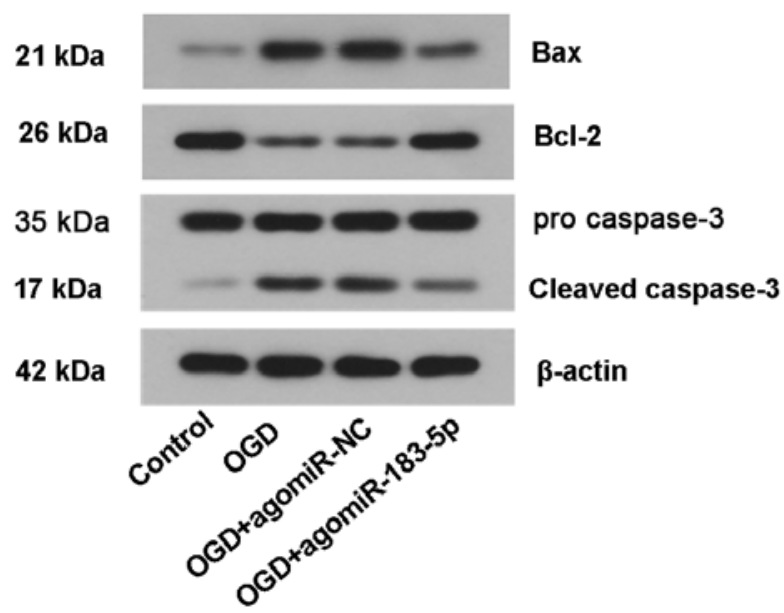

G

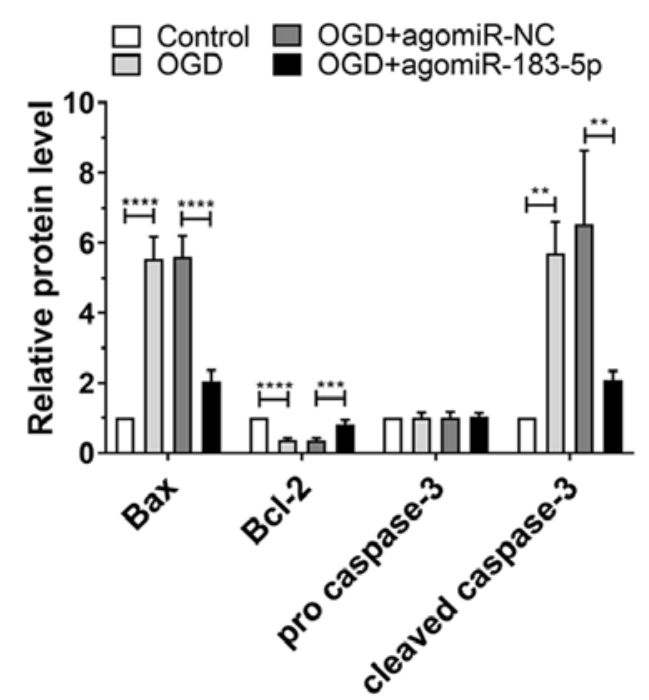

Figure 3. Effects of miR-183-5p on OGD-induced ischemic injury in N2A cells. N2A cells were transfected with agomiR-NC or agomiR-183-5p before OGD. (A) Relative expression levels of miR-183-5p in N2A cells were detected by reverse transcription-quantitative PCR. (B) Relative expression levels of miR-183-5p in the ischemic penumbra after transfection with agomiR-183-5p. (C) Survival of N2A cells assessed by Cell Counting Kit-8 assay. (D and E) Apoptotic N2A cells evaluated by flow cytometry assay and quantitative analysis. (F and G) Relative protein expression levels of the Bax, Bcl-2 and cleaved caspase-3 in N2A cells detected by western blot and densitometry analysis. Data are expressed as the mean $\pm \mathrm{SD}(\mathrm{n}=3) .{ }^{* *} \mathrm{P}<0.01,{ }^{* * *} \mathrm{P}<0.001$ and ${ }^{* * * *} \mathrm{P}<0.0001$. N2A, Neuro-2A; OGD, oxygen-glucose deprivation; miR, microRNA; NC, negative control; OD, optical density; PI, propidium iodide.

overexpression plasmid markedly increased the PTEN levels compared with those in cells transfected with the empty vector $(\mathrm{P}<0.05 ;$ Fig. 4H). The survival of N2A cells exposed to OGD was subsequently analyzed by CCK-8 and flow cytometry assays. As presented in Fig. 4I, N2A cell viability was significantly decreased after transfection with PTEN-overexpression plasmid compared with the control vector plasmids $(\mathrm{P}<0.05)$. In addition, the apoptotic rate of OGD-treated N2A cells was significantly increased following co-transfection with the PTEN overexpression plasmid and agomiR-183-5p compared with that of cells transfected with agomiR-183-5p and the control vector $(\mathrm{P}<0.05$; Fig. $4 \mathrm{~J}$ and $\mathrm{K})$. These results suggested that miR-183-5p inhibited apoptosis and increased cell viability by negatively regulating PTEN in N2A cells.

\section{Discussion}

A mouse model of the cerebral $I / R$ is a commonly used experimental method for investigating the pathophysiology of ischemia-induced brain injury (25). C57BL/6 mice are an extensively used strain in cerebral I/R experiments since they can be easily genetically manipulated (26). The current study 
A miR-183-5p 3'-UCACUUAAGAUGGUCACGGUAU-5' WT-PTEN-site1 5'-CUUGGACAUUUUUGCCAUA-3, MUT-PTEN-site1 5'-CUUGGACAUUUUUCGGUAU-3'

C
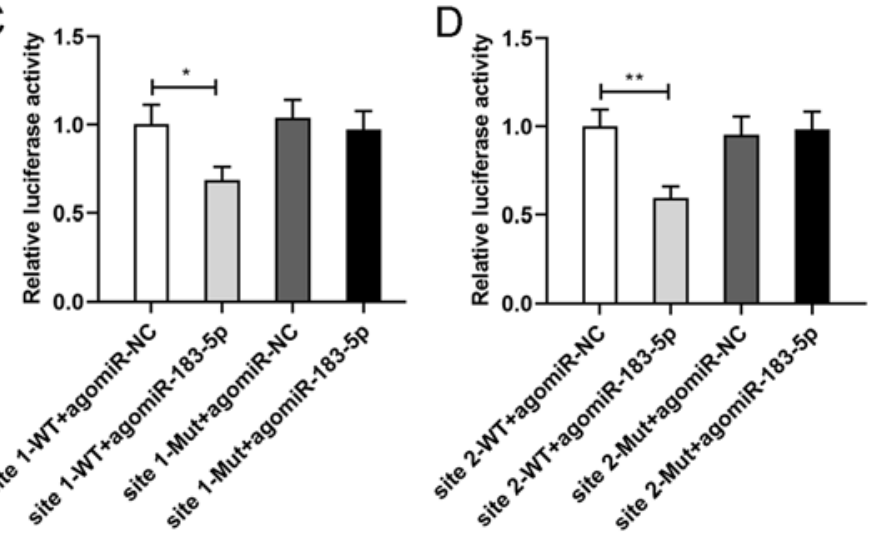

$\mathrm{H}$

G

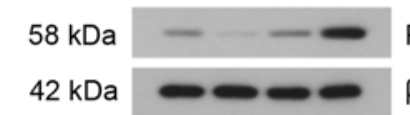

PTEN
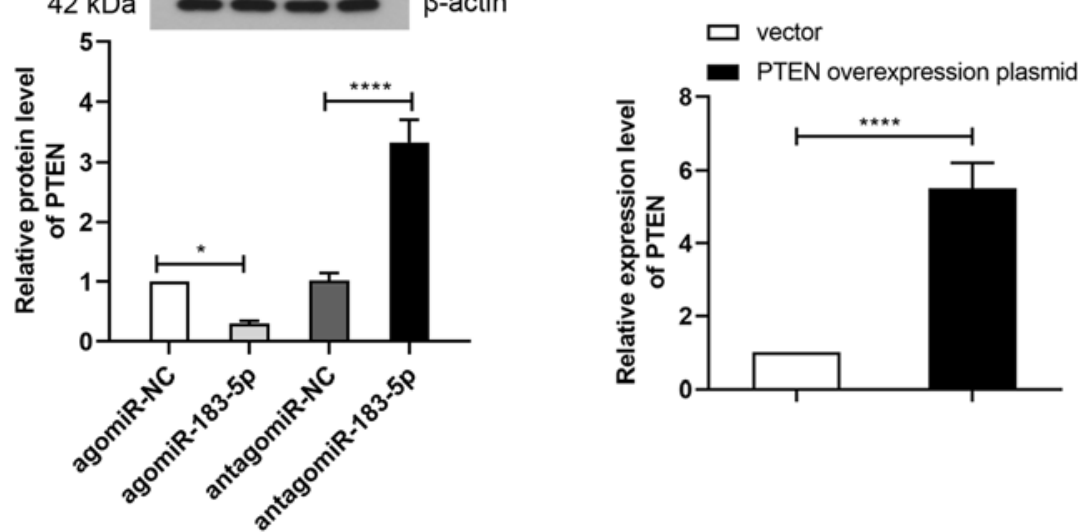

B miR-183-5p 3'-UCACUUAAGAUGGUCACGGUAU-5' miR-183-5p 3'-UCACUUAAGAUGGUCACGGUAU-5'
WT-PTEN-site2 5'-GGAGACAUUAACUGCCAUA-3, MUT-PTEN-site2 5'-GGAGACAUUAACUCGGUAU-3'
$\mathrm{E}$

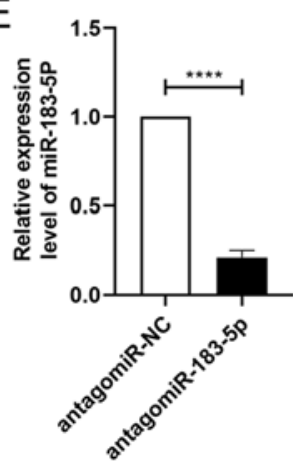

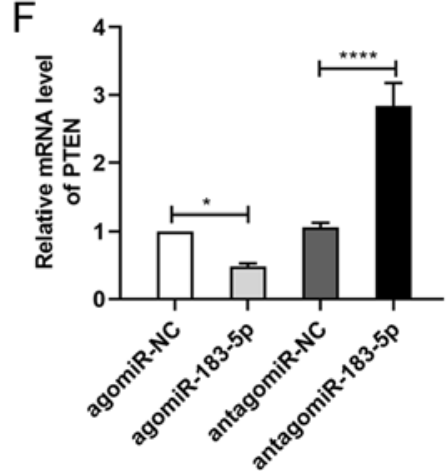

I

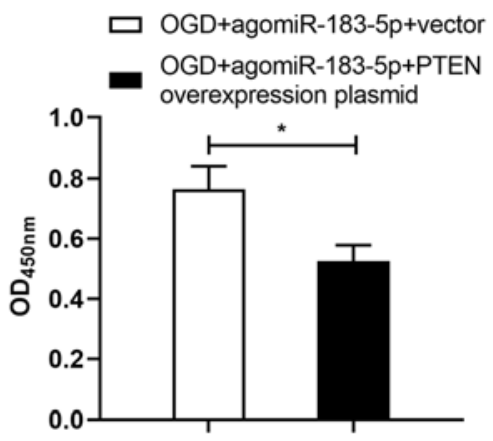

$\mathrm{J}$

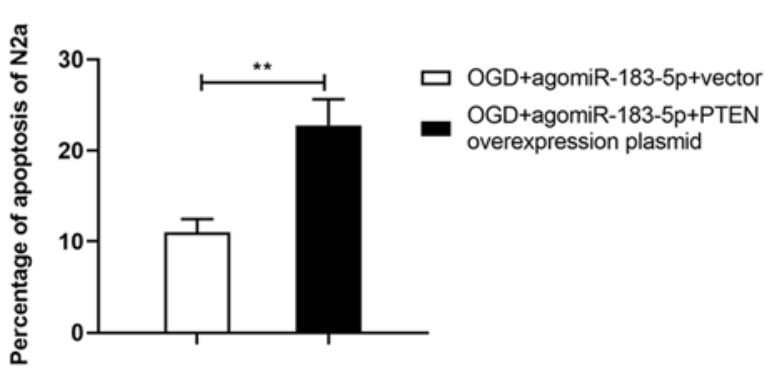

K

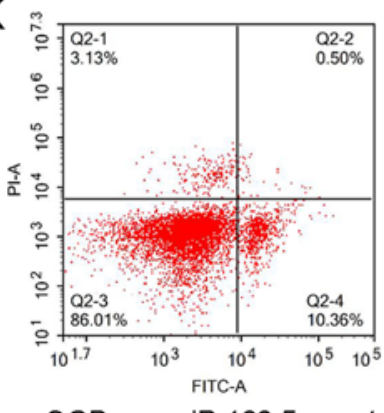

OGD+agomiR-183-5p+vector

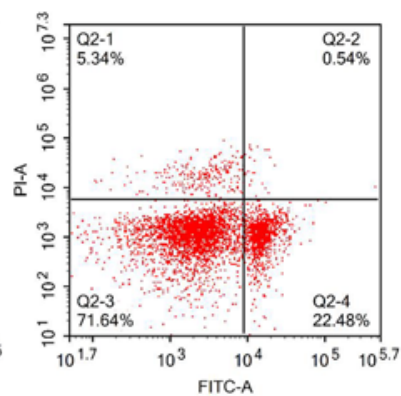

OGD+agomiR-183-5p+

PTEN overexpression plasmid

Figure 4. PTEN is the target of miR-183-5p. (A and B) Conserved miR-183-5p binding sites in the PTEN 3'UTR (A) site 1 and (B) site 2. (C and D) PTEN 3'-UTR sites 1 and 2 mediate the miR-183-5p-mediated control of PTEN expression. (E) The relative expression levels of miR-183-5p in N2A cells after transfection with antagomiR-183-5p. (F) The relative mRNA expression levels of PTEN in N2A cells detected by reverse transcription-quantitative PCR. (G) The relative protein expression levels of PTEN in N2A cells detected by western blot and grayscale analysis. $(\mathrm{H}) \mathrm{The}$ relative expression levels of miR-183-5p in N2A cells after transfection with the PTEN overexpression plasmid. (I) Survival N2A cells assessed after PTEN overexpression by Cell Counting Kit-8 assay. $(\mathrm{J}$ and $\mathrm{K}$ ) Apoptotic N2A cells evaluated after PTEN overexpression by flow cytometry assay and quantitative analysis. Data are expressed as the mean \pm SD $(\mathrm{n}=3) .{ }^{*} \mathrm{P}<0.05,{ }^{* *} \mathrm{P}<0.01$ and ${ }^{* * * * *} \mathrm{P}<0.0001$. UTR, untranslated region; WT, wild-type; MUT, mutant; N2A, Neuro-2A; PTEN, phosphatase and tensin homolog; miR, microRNA; NC, negative control; OD, optical density.

evaluated the role and regulatory mechanism of miR-183-5p in cerebral ischemia. The expression of miR-183-5p was decreased and brain damage was increased in mice following ischemia model establishment compared with those in the sham group. Similar results were observed in an ischemia model in N2A cells, where miR-183-5p expression was reduced and apoptosis was increased compared with those in untreated cells. In further experiments, following transfection with agomiR-183-5p, cerebral ischemia injury and apoptosis levels were reduced compared with those in the MCAO + 
agomiR-NC group. In addition, PTEN was identified as a direct target of miR-183-5p, and the expression of PTEN was negatively regulated by miR-183-5p. Following PTEN overexpression, the neuroprotective effects of miR-183-5p were reduced.

A previous study has demonstrated that the majority of neurons in the area of ischemic injury undergo necrosis or apoptosis (20). In contrast to necrosis, apoptosis is a method of programmed cell death that is regulated by a variety of molecular mechanisms (27). Therefore, apoptotic cells can be salvaged by treatment, with neuronal damage also being alleviated. Yin et al (28) have demonstrated that inhibition of miR-497 attenuated apoptosis and neuronal damage by enhancing the expression of certain antiapoptotic proteins, including $\mathrm{Bcl}-2$ and $\mathrm{Bcl}-2$-family protein (Bcl-w). Zhang et al (29) have revealed that following transfection with miR-378 agomir, upregulation of miR-378 attenuated ischemic injury by negatively regulating the apoptosis-associated protein caspase-3. Although the regulation of miR-183 expression is considered to be effective for treating acute myocardial infarction or liver damage caused by ischemia $(12,13)$, reports detailing miR-183 treatment in cerebral ischemia are unknown. As a member of the miR-183 gene cluster, the normal expression of miR-183-5p is important for nervous system function (30). A previous study has revealed that 17 miRNAs, including miR-183-5p, were downregulated in infarcted brain areas following focal cerebral ischemia in rats according to microRNA sequencing compared with healthy tissues (31). The results of the present study demonstrated decreased levels of miR-183-5p in MCAO models of cerebral ischemia injury and OGD-treated N2A cells compared with those in the respective control groups.

Brain injury caused by cerebral ischemia can lead to neurological deficit symptoms and local infarction (29). A previous study has demonstrated that neurological scores and cerebral edema can reflect the degree of brain damage (32). Additionally, neuronal damage and infarct size may visually indicate the extent of brain damage (33). MCAO causes motor and cognitive impairments reflecting the dysfunction in the frontal and parietal lobes (34). To assess the neurological dysfunction and neuronal damage in mice following MACO, agomiR-183-5p was injected intracerebroventricularly into the experimental group mice; injection site was located in the cerebral cortex around the frontal and parietal lobes. A previous report has detailed the intracerebral injection of miR-183-5p mimics and its efficient amelioration of neuropathic pain by targeting the tandem-pore-domain potassium channel TREK-1 (35). In congruence with these results, the neurological scores and brain edema assay results of the current study, along with TTC, Nissl and FJB staining, revealed that the transfection with agomiR-183-5p alleviated brain injury by reducing neuronal injury, brain water content and brain infarction size. Similarly, in vitro experiment results demonstrated that N2A cells underwent apoptosis following exposure to OGD. AgomiR-183-5p inhibited OGD-induced N2A cell apoptosis and increased cell viability. Extrinsic and intrinsic pathways are recognized to be involved in apoptosis, which relies on alterations in the expression levels of $\mathrm{Bcl}-2$ and caspase family proteins, including Bcl-2, Bcl-xl, Bax and caspase-3 $(36,37)$. The results of the present study demonstrated that following transfection with agomiR-183-5p, apoptosis was decreased, and the cell survival rate was increased compared with those in the agomiR-NC-transfected group. In addition, the expression levels of Bax and cleaved caspase- 3 were decreased, whereas those of Bcl-2 and Bcl-xl were increased in the agomiR-183-5p group compared with those in the agomiR-NC group. As genes that inhibit apoptosis, Bcl-2 and Bcl-xl attenuate the role of the pro-apoptotic gene Bax; therefore, in most cells, apoptosis is controlled by $\mathrm{Bcl}-2$ family proteins (38). In the current study, transfection with agomiR-183-5p promoted $\mathrm{Bcl}-2$ expression, which in turn led to a decrease in Bax expression, thereby maintaining a high cell survival rate. Similarly, as a pro-apoptotic protein (39), caspase-3 expression was decreased following transfection with agomiR-183-5p in the present study. This result was in agreement with a previous report, in which treatment of glabridin decreased caspase-3 transcriptional activity and inhibited staurosporine-induced rat cortical neuronal apoptosis (40). Therefore, the results of the present study suggested that miR-183-5p may regulate apoptosis, as it is associated with the expression of Bcl-2, Bax and cleaved caspase- 3 expression.

PTEN is a tumor suppressor gene with phosphatase activity that modulates cell survival, apoptosis and metabolism by negatively regulating the PI3K/Akt signaling pathway (41). Increasing studies have confirmed that the inhibition of PTEN exerts neuroprotective effects in cerebral ischemic injury $(42,43)$. In MCAO and OGD models, it has been determined that PTEN is highly expressed and contributes to the development of ischemic stroke through the PI3K/Akt pathway $(42,43)$. The bioinformatics analysis in the present study revealed that PTEN was a target of miR-183-5p. A previous study has also revealed that PTEN is a target of miR-130a and can reverse the protective effects of miR-130a on cerebral I/R damage in vivo and vitro (44), indicating that PTEN serves an important role in the response to ischemia. Similarly, the results of the present study demonstrated that miR-183-5p and PTEN formed a negative feedback loop, and that PTEN overexpression inhibited the protective effects exerted by miR-183-5p on cell viability, which in turn increased apoptosis. The current results only suggested that miR-183-5p decreased cerebral ischemic injury by targeting PTEN; however, PTEN expression and the effects of agomiR-183-5p on PTEN activation after MCAO or OGD are not clear and require further study.

In conclusion, the present study primarily clarified the function and mechanism of miR-183-5p in cerebral ischemia. Transfection with agomiR-183-5p inhibited apoptosis and increased cell viability by negatively regulating the expression of PTEN, thus mitigating cerebral ischemic injury. These findings may contribute to the further understanding of the mechanism of cerebral ischemia and may provide novel insights into ischemic injury treatment.

\section{Acknowledgements}

Not applicable.

\section{Funding}

No funding was received. 


\section{Availability of data and materials}

The datasets used and/or analyzed during the current study are available from the corresponding author on reasonable request.

\section{Authors' contributions}

JL conceived and designed the study. LZ, SL, SZ, JY and $\mathrm{XF}$ prepared the materials, performed the experiments, collected and analyzed the data. LZ drafted the manuscript. $\mathrm{XZ}$ performed the supplementary experiments and revised the manuscript. All authors read and approved the final manuscript.

\section{Ethics approval and consent to participate}

All the procedures in the animal experiments followed the guide for the care and use of laboratory animals, and this study was approved by Institutional Animal Care and Use Committee of Binzhou People's Hospital (approval no. LYP200).

\section{Patient consent for publication}

Not applicable.

\section{Competing interests}

The authors declare that they have no competing interests.

\section{References}

1. Singh V, Roth S, Veltkamp R and Liesz A: HMGB1 as a key mediator of immune mechanisms in ischemic stroke. Antioxid Redox Signal 24: 635-651, 2016.

2. Zerna $\mathbf{C}$, Hegedus $\mathbf{J}$ and Hill MD: Evolving treatments for acute ischemic stroke. Circ Res 118: 1425-1442, 2016.

3. Lakhan SE, Kirchgessner A and Hofer M: Inflammatory mechanisms in ischemic stroke: Therapeutic approaches. J Transl Med 7: 97, 2009.

4. Fonarow GC, Smith EE, Saver JL, Reeves MJ, Bhatt DL, Grau-Sepulveda MV, Olson DM, Hernandez AF, Peterson ED and Schwamm LH: Timeliness of tissue-type plasminogen activator therapy in acute ischemic stroke: Patient characteristics, hospital factors, and outcomes associated with door-to-needle times within 60 minutes. Circulation 123: 750-758, 2011.

5. Rami A and Kogel D: Apoptosis meets autophagy-like cell death in the ischemic penumbra: Two sides of the same coin? Autophagy 4: 422-426, 2008.

6. Hammond S: An overview of microRNAs. Adv Drug Deliv Rev 87: 3-14, 2015.

7. Li G, Morris-Blanco KC, Lopez MS, Yang T, Zhao H, Vemuganti R and Luo Y: Impact of microRNAs on ischemic stroke: From preto post-disease. Prog Neurobiol 163-164: 59-78, 2018.

8. Birch D, Britt BC, Dukes SC, Kessler JA and Dizon ML: MicroRNAs participate in the murine oligodendroglial response to perinatal hypoxia-ischemia. Pediatr Res 76: 334-340, 2014.

9. Majdi A, Mahmoudi J, Sadigh-Eteghad S, Farhoudi M and Shotorbani SS: The interplay of microRNAs and post-ischemic glutamate excitotoxicity: An emergent research field in stroke medicine. Neurol Sci 37: 1765-1771, 2016.

10. Chang L, Zhang W, Shi S, Peng Y, Wang D, Zhang L and Zhang J: microRNA-195 attenuates neuronal apoptosis in rats with ischemic stroke through inhibiting KLF5-mediated activation of the JNK signaling pathway. Mol Med 26: 31, 2020.

11. Pierce ML, Weston MD, Fritzsch B, Gabel HW, Ruvkun G and Soukup GA: Microrna-183 family conservation and ciliated neurosensory organ expression. Evol Dev 10: 106-113, 2008.

12. Lin HC, Liu SY, Yen EY, Li TK and Lai IR: microRNA-183 mediates protective postconditioning of the liver by repressing Apaf-1. Antioxid Redox Signal 26: 583-597, 2017.
13. Gong $\mathrm{L}, \mathrm{Xu} \mathrm{H}$, Chang $\mathrm{H}$, Tong $\mathrm{Y}$, Zhang $\mathrm{T}$ and Guo G: Knockdown of long non-coding RNA MEG3 protects H9c2 cells from hypoxia-induced injury by targeting microRNA-183. J Cell Biochem 119: 1429-1440, 2018.

14. Garcia-Junco-Clemente P and Golshani P: PTEN: A master regulator of neuronal structure, function, and plasticity. Commun Integr Biol 7: e28358, 2014.

15. Miao SY, Miao SM, Cui RT, Yu AL and Miao ZJ: SETD5-AS1 stimulates neuron death in stroke via promoting PTEN expression. Eur Rev Med Pharmacol Sci 22: 6035-6041, 2018.

16. Sarver AL, Li L and Subramanian S: MicroRNA miR-183 functions as an oncogene by targeting the transcription factor EGR1 and promoting tumor cell migration. Cancer Res 70: 9570-9580, 2010.

17. Thiel J, Alter C, Luppus S, Eckstein A, Tan S, Führer D, Pastille E, Westendorf AM, Buer J and Hansen W: MicroRNA-183 and microRNA-96 are associated with autoimmune responses by regulating T cell activation. J Autoimmun 96: 94-103, 2019.

18. Guo D, Ma J, Li T and Yan L: Up-regulation of miR-122 protects against neuronal cell death in ischemic stroke through the heat shock protein 70-dependent NF-kappaB pathway by targeting FOXO3. Exp Cell Res 369: 34-42, 2018.

19. Ni J, Wang X, Chen S, Liu H, Wang Y, Xu X, Cheng J, Jia J and Zhen X: MicroRNA let-7c-5p protects against cerebral ischemia injury via mechanisms involving the inhibition of microglia activation. Brain Behav Immun 49: 75-85, 2015.

20. Chen J, Sanberg PR, Li Y, Wang L, Lu M, Willing AE, Sanchez-Ramos $\mathbf{J}$ and Chopp M: Intravenous administration of human umbilical cord blood reduces behavioral deficits after stroke in rats. Stroke 32: 2682-2688, 2001.

21. Yoon S, Woo SU, Kang JH, Kim K, Kwon MH, Park S, Shin HJ, Gwak HS and Chwae YJ: STAT3 transcriptional factor activated by reactive oxygen species induces IL6 in starvation-induced autophagy of cancer cells. Autophagy 6: 1125-1138, 2010.

22. Wang X, Wang S, Wang J, Guo H, Dong Z, Chai L, Hu L, Zhang Y, Wang $\mathrm{H}$ and Chen L: Neuroprotective effect of xueshuantong for injection (Lyophilized) in transient and permanent rat cerebral ischemia model. Evid Based Complement Alternat Med 2015: 134685, 2015.

23. Gong SJ, Chen LY, Zhang M, Gong JX, Ma YX, Zhang JM, Wang YJ, Hu YY, Sun XC, Li WB and Zhang Y: Intermittent hypobaric hypoxia preconditioning induced brain ischemic tolerance by up-regulating glial glutamate transporter-1 in rats. Neurochem Res 37: 527-537, 2012.

24. Livak KJ and Schmittgen TD: Analysis of relative gene expression data using real-time quantitative PCR and the 2(-Delta Delta C(T)) method. Methods 25: 402-408, 2001.

25. Woodruff TM, Thundyil J, Tang SC, Sobey CG, Taylor SM and Arumugam TV: Pathophysiology, treatment, and animal and cellular models of human ischemic stroke. Mol Neurodegener 6: 11, 2011.

26. Yonekura I, Kawahara N, Nakatomi H, Furuya K and Kirino T: A model of global cerebral ischemia in C 57 BL/6 mice. J Cereb Blood Flow Metab 24: 151-158, 2004.

27. Doyle KP, Simon RP and Stenzelpoore MP: Mechanisms of ischemic brain damage Neuropharmacology 55: 310-318, 2008.

28. Yin KJ, Deng Z, Huang H, Hamblin M, Xie C, Zhang J and Chen YE: miR-497 regulates neuronal death in mouse brain after transient focal cerebral ischemia. Neurobiol Dis 38: 17-26, 2010.

29. Zhang N, Zhong J, Han S, Li Y, Yin Y and Li J: MicroRNA-378 alleviates cerebral ischemic injury by negatively regulating apoptosis executioner caspase-3. Int J Mol Sci 17: 1427, 2016.

30. Banks SA, Pierce ML and Soukup GA: Sensational MicroRNAs: Neurosensory roles of the MicroRNA-183 family. Mol Neurobiol 57: 358-371, 2020.

31. Duan X, Gan J, Peng DY, Bao Q, Xiao L, Wei L and Wu J: Identification and functional analysis of microRNAs in rats following focal cerebral ischemia injury. Mol Med Rep 19: 4175-4184, 2019.

32. Ye Y, Jin T, Zhang X, Zeng Z, Ye B, Wang J, Zhong Y, Xiong X and $\mathrm{Gu} \mathrm{L}$ : Meisoindigo protects against focal cerebral ischemia-reperfusion injury by inhibiting NLRP3 inflammasome activation and regulating microglia/macrophage polarization via TLR4/NF- $\mathrm{B}$ signaling pathway. Front Cell Neurosci 13: 553, 2019.

33. Park SJ, Nam KW, Lee HJ, Cho EY, Koo U and Mar W: Neuroprotective effects of an alkaloid-free ethyl acetate extract from the root of Sophora flavescens Ait. against focal cerebral ischemia in rats. Phytomedicine 16: 1042-1051, 2009. 
34. Truong DT, Venna VR, McCullough LD and Fitch RH: Deficits in auditory, cognitive, and motor processing following reversible middle cerebral artery occlusion in mice. Exp Neurol 238: 114-121, 2012.

35. Shi DN, Yuan YT, Ye D, Kang LM, Wen J and Chen HP. MiR-183-5p alleviates chronic constriction injury-induced neuropathic pain through inhibition of TREK-1. Neurochem Res 43: 1143-1149, 2018.

36. Pilchova I, Klacanova K, Chomova M, Tatarkova Z, Dobrota D and Racay P: Possible contribution of proteins of Bcl-2 family in neuronal death following transient global brain ischemia. Cell Mol Neurobiol 35: 23-31, 2015.

37. Datta A, Sarmah D, Mounica L, Kaur H, Kesharwani R, Verma G, Veeresh P, Kotian V, Kalia K, Borah A, et al: Cell death pathways in ischemic stroke and targeted pharmacotherapy. Transl Stroke Res: 26 Mar, 2020 (Epub ahead of print)

38. Cory S, Huang DC and Adams JM: The Bcl-2 family: Roles in cell survival and oncogenesis. Oncogene 22: 8590-8607, 2003.

39. Massieu L, Moran J and Christen Y: Effect of Ginkgo biloba (EGb 761) on staurosporine-induced neuronal death and caspase activity in cortical cultured neurons. Brain Res 1002: 76-85, 2004

40. Yu XQ, Xue CC, Zhou ZW, Li CG, Du YM, Liang J and Zhou SF: In vitro and in vivo neuroprotective effect and mechanisms of glabridin, a major active isoflavan from Glycyrrhiza glabra (licorice). Life Sci 82: 68-78, 2008.
41. Oudit GY, Sun H, Kerfant BG, Crackower MA, Penninger JM and Backx PH: The role of phosphoinositide-3 kinase and PTEN in cardiovascular physiology and disease. J Mol Cell Cardiol 37: 449-471, 2004

42. Xing Y, Wang MM, Feng YS, Dong F and Zhang F: Possible involvement of PTEN signaling pathway in the anti-apoptotic effect of electroacupuncture following ischemic stroke in rats. Cell Mol Neurobiol 38: 1453-1463, 2018.

43. Zhao D, Chen J, Zhang Y, Liao HB, Zhang ZF, Zhuang Y, Pan MX, Tang JC, Liu R, Lei Y, et al: Glycine confers neuroprotection through PTEN/AKT signal pathway in experimental intracerebral hemorrhage. Biochem Biophys Res Commun 501: 85-91, 2018

44. Zheng T, Shi Y, Zhang J, Peng J, Zhang X, Chen K, Chen Y and Liu L: MiR-130a exerts neuroprotective effects against ischemic stroke through PTEN/PI3K/AKT pathway. Biomed Pharmacother 117: 109117, 2019.

This work is licensed under a Creative Commons Attribution-NonCommercial-NoDerivatives 4.0 International (CC BY-NC-ND 4.0) License. 